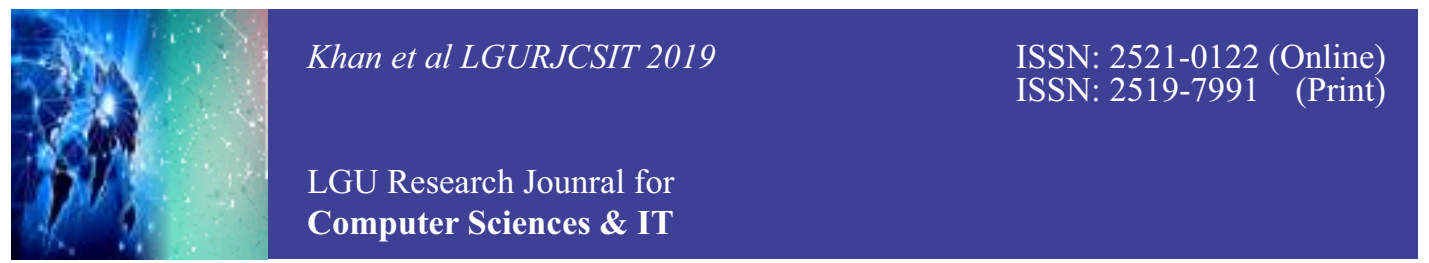

Vol. 3 Issue 2, April - June 2019

\title{
Routing Protocols Evaluation Review in Simple and Cloud Environment
}

Rafaqat Alam Khan, Muhammad Harith Javed

Lahore Garrison University, DHA Campus, Lahore, Pakistan

\section{Abstract:}

In the field of information technology there are many computer jargons like cloud computing Ad-hoc, Software Define Network (SDN), network function virtualization (NFV), and virtual machine (VM), etc. This review paper is basically a blend of brief study and review of many routing protocols used for Mobile ad hoc Networks (MANET) in the cloud as well as in simple network environment i.e. without cloud computing. This paper would also suggest the different challenges that are facing in cloud computing. The description of the different network simulators used in networking like NS2 tool, Opnet and Cisco packet tracer. The different metrics that are used in the networking are briefly explained. MANET is a group of wireless nodes that do not need centralized controlling entity as it rapidly moves changes and forms networks to the nearest networking nodes.

Keywords: $\quad$ Protocol, MANET, SDN, Cloud Computing

\section{Introduction}

The very basic definition of the protocol is the set of rules through in which two electronic nodes are connected. Thus there are many rules governing the routing of the connection from the source to the destination. This research review analysis of different routing protocol which is used both in simple and cloud networking environment.

The simple network comprises a computer system, routers, switches, cables for connection, bridges, etc. But the latest trend is the advancement of cloud computing. It is the process of moving the whole of the computing including networking to the virtual world. The virtual world terminology means here that in the computer networking field have to provide the opportunity to make virtual computers, including virtual hard disk, RAM, Networks even including router, switches, and cables, etc. This research is compromised by analyzing some of the routing protocol that is used in networking simple and in a cloud environment [1-7].

\section{Cloud Computing in Brief}

There are many definitions of cloud computing according to the perception people have. The simplest definition of cloud computing is the processing of computer operation from normal personal computers to the data centers and to the internet. This processing of computer operation has enabled the hardware/software requirements of the users, available as service over the internet. The users are the cloud tenants who acquire these services as per their demand and payed according to the usage of the computer they have acquired. Thus, we can say that cloud services are featured by on-demand self-service, broad network access, resource pooling, elasticity, scalability and lastly services according to the requirements. There are 3 types of cloud services namely Software as a Service (SAAS), Platform as a Service (PAAS), Infrastructure as a service (IAAS).

\subsection{Software as a Service (SAAS):}

In this type of Cloud computing service (SAAS), the cloud tenants have access to the cloud supporting applications running on the 
available data centers. This type of cloud service has limited access to the users; basically, it is fixed to only the application configuration settings. Google apps, Salesforce, Cisco WebEx etc are the different examples that work under this category. SAAS along with different security challenges in both normal and cloud environment are briefly discussed and they have also suggested a model tackle those security issues [1-2].

\subsection{Platform as a Service (PAAS):}

In this type of cloud service (PAAS), the tenant can deploy their own application on the cloud using programming languages and tools supported by their available data centers. Cloud tenants have control over the developed applications and their hosting environment. Amazon web services (AWS), elastic beanstalk, Windows Azure, Google apps, etc are the examples that works under this category of cloud computing. The different security issues related to each of these cloud computing services such as SAAS, PAAS, and IAAS are discussed in detail [3-4].

\subsection{Infrastructure as a Service (IAAS):}

This type of cloud service (IAAS) provide services to the users that can pool the resources according to their need. These resources include storage, Network, and basic computing. These resources are acquired by the creation of virtual machines. Users have control over the operating system like DSL, Centos, Ubuntu, Storage, Deployed apps, Limited control over the network (as provider allows access to the administrator to control networking resources) [3]. In this type of service, the user is enabled to incorporate their network on the cloud. Amazon web services (AWS), Cisco Meta Pod, Microsoft Azure, etc are the examples that are working under this environment of cloud computing. The comparative analyses of different IAAS cloud environment are discussed in detail by the Sonia Shahzadi et all .

\subsection{Networking in Cloud}

In cloud system, the networking is almost same as that of normal computer networking but the difference exists in one part that in cloud computing the nodes (Router, Switches, etc) and the cable connections can be virtualized. This enables the users to deploy any type of virtual machine creating running on a hypervisor (ESXi, Hyper V, V Sphere, etc) and the virtual cables are used to connect these hosts. This whole process is termed as network function virtualization. It discusses the different challenges and opportunities for innovation in network function virtualization in detail. V. Gopalakrishnan et all 2015 .

\subsection{Mobile Ad Hoc Networks in Brief}

This type of networks compromised of a group of wireless mobile nodes which automatically create the network while in a move to the nearest network setup available. WiFi is a good example that works under MANET. In this type of network, each node works as a router. This research gives a brief review of some routing protocols used in MANET both in simple and cloud environment. Sharma et al. 2013 discuss a brief survey related to MANET for the enhancement of performance using data cache schemes. Some basic routing protocols that work under the MANET are as follows [8].

\subsubsection{Proactive}

In this type, the routing table is maintained and has the predefined address of the destination for the data in transmission. Thus in this type, the packet forwarding process is fast but the routing cost is high because of the predefinition of all routes in the network. The discussion related to opportunist data forwarding in MANET using proactive source routing is explained by Mule et al 2016 . A review of proactive in MANET is discussed in . The different examples of proactive routing protocols are distance vector, Destination Sequence Distance Vector (DSDV) Fisheye State Routing (FSR), Optimized Link State Routing (OLSR) etc [11-16].

\subsubsection{Reactive}

Reactive protocols are those protocols having high latency but low routing overhead. In this type of routing protocol, the routing table isn't maintained instead of that, flooding algorithm is used to send the data packets. So when the connection is established in this type of routing protocols the data is sent to the immediate neighbors till it reaches the destination. Ad-Hoc on Demand Distance Vector Routing (AODV) and Dynamic Source Routing (DSR) are examples of reactive 
protocols. The traffic density effects on the performance of the reactive routing protocol are discussed in detail in. They have suggested AODV protocol for the mobile ad-hoc network through power optimizing protocol .

\subsubsection{Hybrid}

Hybrid protocols have the pros of both the protocols i.e. Proactive and Reactive protocols. Thus in this type of routing protocols, there is a lesser cost for routing and route table maintenance. Temporally Ordered Routing Algorithm (TORA) and Enhanced Interior Gateway Routing Protocol (EIGRP) are the examples that work under hybrid routing protocols $[19,20]$.

\subsection{Dynamic Source Routing}

Path learning of the route in the network is involved here. To send data packets source node sends a route request in the entire network if there is no route cached. Route information is added and maintained if the node is an intermediate node. DSR are similar to the AODV as packet transfer is on demand. Route request and route reply messages are used to intercommunicate between the nodes [21-22].

\subsection{Temporally Ordered Routing Algorithm}

This protocol with the path learning adds additional features like route deletion, discovery, and maintenance. This has eliminated the cycles or loops in the routes. Hence routing overhead is reduced. This process in algorithm study is called directed acyclic graphs. All routes are found but the shortest route is selected and all nodes making a cycle are deleted from the cache by sending CLR packets [23].

\subsection{Low Energy Adaptive Clustering Hierarchy}

This protocol involves the clustering of the nodes. It comprises low powered sensors on which energy is equally circularized. Thus, the node has to do minimal work. This involves setting up phase when the cluster head is chosen and steady phase when the cluster is maintained during data packet transmission. Cluster head collects data and sends it to the base station. The job of the head is in rotation thus LEACH is dynamic [24-25].
There are some protocols of LEACH that works under the cloud environment. The purpose of those protocols under the cloud environment is used for data encryption, communication, data transmission, remote login, etc. The different types of cloud routing protocols are explained as below [26].

\subsection{Gossip Protocols}

Gossip protocols are also termed as epidemic protocols. It is basically used for communication and anti-entropy, used here as a data repairing when data becomes disordered. These protocols use dissemination protocols to spread information which in turn work through the flooding agent in the network. These are also used for failure detection, messaging (using TCP to wrap data) and monitoring the data with a separate mechanism [27-29].

\subsection{Connectionless Network Protocols}

This works on the 3rd layer of the OSI model. It comprises the mechanism for the data fragmentation like data unit identification, length of data, offset address, etc. The address size of connection-less network protocols is 20 bytes rather than 4 bytes [30-32].

\subsection{Internet Group Management Protocols}

These like other management protocols such as Internet Control Message Protocol (ICMP) work on the network layer. The functionality of this protocol is to multicast the data over the nodes in the network through a router communication protocol. Video streaming and gaming on the cloud are examples of IGMP [33-35].

\subsection{Secure Shell Routing Protocol}

Secure Shell is a cryptographic protocol that allows secure remote login. The decryption algorithm is placed in the remote server [36-37]. This protocol is the potential replacement of the TELNET and having two version SSH-1 and SSH-2 [38].

\subsection{Coverage Enhanced Routing Protocols}

Data packets are lost when the new data is incoming via the switch. This protocol is used to eliminate this problem to some extent. It 
handles the data packets on the data link layer with lower cost for storing the data packets [39].

\subsection{Extensible Messaging and Presence Protocol}

It is a file transfer protocol used in cloud computing. Microsoft published Extensible Messaging and Presence Protocol (XMPP) interface for messaging. Other uses of this protocol are for publishing, subscriber system videos, etc [40-41].

\subsection{Advanced Message Queuing Protocol}

This protocol works on the application layer and provides guaranteed message delivery. It is primarily used in the cloud, but now it's being used in Red hat, Microsoft, Apache, etc. Here messages and other information are routed in Peer to Peer (P2P) over the cloud. The format of the data sent over the cloud network is given by the wireless protocol [42].

\subsection{Media Transfer Protocol}

The MTP is used for the transfer to and from of the audio, media, metadata files over the cloud by the use of the P2P protocol. Downloading of media files are done through this protocol [43].

\section{Evaluation/Simulation Tools}

\subsection{OPNET}

This is basically the software in which we can simulate any type on the network on the basis of performance and analyze their behavior. Here we can create our own network topology and evaluate it. It has many built-in project scenarios that can be imported or even duplicated. It is also open-source software. Protocols can be modeled here and any hardware architecture can be validated here virtually [4445].

\section{2. $\quad N S 2$}

Network simulator has variants ranging from NS 1-4. It's a name given to these discrete networks. Simulations here are used for research and teaching purposes. This has a topology definition, Model creation, Node/link configuration, Execution, Performance analysis, and graphical views [46-48].

\subsection{CISCO Packet Tracer}

This model is primarily for the Cisco network infrastructure as it was developed by Cisco systems. This software is a cross-platform simulator that allows a user to simulate and configure the Cisco router and switches using command-line interface. Here drag and drop process is used as an interface for the simulation [49-51].

\subsection{Evaluation/Simulation Parameters}

- Number of nodes

- Simulation time

- Simulation model

- MAC type

- Traffic source

- Packet size

- Area

- Application

- Node energy

- Node speed

- Pause time

\subsection{Evaluation/Simulation Metrics}

The metrics used for evaluation are mentioned as follows:

- Throughput

- End to end delay

- Jitter

- Packet delay ratio

\subsection{Evaluation/Simulation results}

The results of the simulation would be as per the metrics. Through simulation, one can figure out which protocol can be further improved and can be applied efficiently either in cloud or in simple network infrastructure.

\section{Challenges in Cloud Computing}

The predominant cloud networking architecture represents the general denominators and modified demand of a cloud. Many significant design parameters such as network topologies. Forwarding protocols and security policies endeavor to provide to all the demands. Finally, it forecloses the optimal usage and suitable administration of the 
network. Customers' displacement from the physical data center would like to have performance close to it even they obtain less cost. For example, they expect that the cloud will allow them to determine bandwidth demands for applications hosted in the cloud. In many solution customers sign up for stringent service level agreements (SLAs), according to the demands of their application. These need to fulfill in cloud the recourses. Some customer demands a large number of switches and routers. For example, there are a number of network devices, load balancer, firewall, and application acceleration. The traffic flow, which is related to all of these demands is to be disjunction. They demand to get at control for the end-users. Traffic specifies and gets at control to the enduser are among the multiple forwarding policies that should be implemented. These policies directly affects the constellation of each router and switches. Modification, demands, and different protocols are challenges. Connectivity between the data center to cater to the vision of a cloud is another challenge.

\section{Conclusion}

Till now no protocol has been fully accepted or rejected. Every protocol has its own pros and cons as per environment, metrics applied and the topology used. Thus, until this cloud environment is a new system and there is space for some new protocol, so it can be concluded that some protocols are better in this situation and some in other. We after the review of above-mentioned protocols propose that some variation should be made or we can make a blend of some protocols to make a new one which would perform both in simple network infrastructure and in the cloud network.

\section{References}

[1] Javier Barabas. IBM Cloud. [Online]. https://www.ibm.com/cloud/saas

[2] Dr.G.Sahoo2, Dr.S.Mehfuz3 Rashmi 1, "Securing Software as a Service Model of Cloud Computing: Issues and Solutions," International Journal on Cloud Computing: Services and Architecture (IJCCSA), vol. 3, no. 4, August 2013.

[3] Javier Barabas. IBM Cloud. [Online]. https://www.ibm.com/blog s/cloud- computing/2014/02/what-is-platform-as-aservice-paas/

[4] Q. M. Shallal and Y. K. Tamandani M. U. Bokhari, "Cloud computing service models: A comparative study," in 3rd International Conference on Computing for Sustainable Global Development, New Delhi, 2016, pp. 890895.

[5] Javier Barabas. IBM Cloud. [Online]. https://www.ibm.com/cloud/infrastructure

[6] "Infrastructure as a Service (IaaS): A Comparative Performance Analysis of OpenSource Cloud Platforms," in 22nd IEEE International Workshop on Computer Aided Modeling and Design of Communication Links and Networks (CAMAD), Lund, Sweden, 2017.

[7] V. Gopalakrishnan, L. Ji and S. Lee B. Han, "Network function virtualization: Challenges and opportunities for innovations," IEEE Communications Magazine, vol. 53, no. 2, pp. 90-97, Feburary 2015.

[8] K. M. Sharma and A. Raut, "Survey on performance enhancement in mobile adhoc networks using data caching schemes," in International Conference on Information Communication and Embedded Systems (ICICES), Chennai, 2013, pp. 95-98.

[9] R. Mule and B. Patil, "Proactive source routing protocol for opportunistic data forwarding in MANETs," in International Conference on Automatic Control and Dynamic Optimization Techniques (ICACDOT), Pune, 2016, pp. 227-232.

[10] S. Shruthi, "Proactive routing protocols for a MANET - A review," in International Conference on I-SMAC (IoT in Social, Mobile, Analytics and Cloud) (I-SMAC), Palladam, 2017,pp. 821-827.

[11] M. Naseem and C. Kumar, "EDSDV: Efficient DSDV routing protocol for MANET," in IEEE International Conference on Computational Intelligence and Computing Research, Enathi, 2013,pp. 1-4.

[12] T. S. Chouhan and R. S. Deshmukh, "Analysis of DSDV, OLSR and AODV Routing Protocols in VANETS Scenario: Using Ns3," in 
International Conference on Computational Intelligence and Communication Networks (CICN), Jabalpur, 2015, pp. 85-89.

[13] N. R. Sivakumar and C. Chelliah, "Simulation and Evaluation of the Performance on Probabilistic Broadcasting in FSR (Fisheye State Routing) Routing Protocol Based on Random Mobility Model in MANET," in Fourth International Conference on Computational Intelligence, Communication Systems and Networks, Phuket, 2012, pp. 371-376.

[14] L. Liu and Z. Chen, "Enhanced fisheye state routing using cross-layer optimization," in Chinese Control and Decision Conference, Guilin, 2009, pp. 4673-4676.

[15] S. Gadekar and S. Kadam, "Secure optimized link state routing (OLSR) protocol against node isolation attack," in IEEE International Conference on Power, Control, Signals and Instrumentation Engineering (ICPCSI), Chennai, 2017, pp. 684-687.

[16] A. Moussaoui, K. Zouaoui and S. Mehamel F. Semchedine, "CRY OLSR: Crypto Optimized Link State Routing for MANET," in 5th International Conference on Multimedia Computing and Systems (ICMCS), Marrakech, 2016, pp. 290-293.

[17] K. Kanchan and S. C. Gupta, "Effects of traffic density on performance of reactive routing protocols," in International Conference on Control, Automation, Robotics and Embedded Systems (CARE), Jabalpur, 2013, pp. 1-6.

[18] V. Bharathi and P. Vijayakumar T. Poongkuzhali, "An optimized power reactive routing based on AODV protocol for Mobile Adhoc network," in International Conference on Recent Trends in Information Technology (ICRTIT), Chennai, Tamil Nadu, 2011, pp. 194199.

[19] S. Thawani and H. Upadhyay, "Securing TORA against Sybil attack in MANETs," in International Conference on Futuristic Trends on Computational Analysis and Knowledge Management , (ABLAZE), Noida, 2016, pp. 475-478.
[20] K. H. P. Widya, A. Fiade and I. R. Julia S. U. Masruroh, "Performance Evaluation DMVPN Using Routing Protocol RIP, OSPF, And EIGRP," in 6th International Conference on Cyber and IT Service Management (CITSM), Parapat, Indonesia, 2018, pp. 1-6.

[21] Z. G. Al-Mekhlafi and R. Hassan, "Evaluation study on routing information protocol and dynamic source routing in Ad-Hoc network," in 7th International Conference on Information Technology in Asia, Kuching, Sarawak, 2011, pp. 1-4.

[22] C. Perkins, E. Belding-Royer, and S Das, "Ad hoc On-Demand Distance Vector (AODV) Routing https://doi.org/10.17487\%2FRFC 3561," IETF, July 2003.

[23] V. Park and S. Corson. IETF. [Online]. https://web.archive.org/web/20070206094306/ http://www3.ietf.org/proceedings/02 mar/ID/draft-ietf-manet-tora-spec-04.txt

[24] W., Chandrakasan, A., and Balakrishnan, $\mathrm{H}$ Heinzelman, "Energy-Efficient Communication Protocols for Wireless Microsensor Networks," in 33rd Hawaaian International Conference on Systems Science (HICSS), Hawaaian, January 2000.

[25] C. Bosquez, F. Palacios and L. Córdoba G. Alvarado, "Low-energy Adaptive Clustering Hierarchy protocol and optimal number of cluster head algorithm in a randomized wireless sensor network deployment," in International Conference on Electrical, Electronics, Communication, Computer, and Optimization Techniques (ICEECCOT), Mysuru, 2017, pp. $1-4$.

[26] C. Bosquez, F. Palacios and L. Córdoba G. Alvarado, "Low-energy Adaptive Clustering Hierarchy protocol and optimal number of cluster head algorithm in a randomized wireless sensor network deployment," in International Conference on Electrical, Electronics, Communication, Computer, and Optimization Techniques (ICEECCOT), Mysuru, 2017, pp. 14.

[27] Alan Demers et al., "Epidemic Algorithms for Replicated Database Maintenance," in Sixth Annual ACM Symposium on Principles of Distributed 
Computing, 87. New York, NY, USA, 1987, pp. 1-12.

[28] H. Kurdi, A. Alromih, A. Alomari, E. Alrogi and S. H. Ahmed L. Altoaimy, "Enhanced Distance-Based Gossip Protocols for Wireless Sensor Networks," in 16th IEEE Annual Consumer Communications \& Networking Conference (CCNC), Las Vegas, NV, USA, 2019,pp. 1-4.

[29] X. Hong and Z. Jie, "Research of a New Messages Dissemination Algorithm Based on Structured Gossip Protocol," in International Conference on Electrical and Control Engineering, wuhan, 2010,pp. 5881-5884.

[30] Section 3: Definition of the Connectionless-Mode Service. (2001, October) ITU-T Recommendation X.213: Open Systems Interconnection - Network service definition, International Telecommunications Union. [Online]. https://www.itu.int/rec/T-RECX.213/en/

[31] S. Muramatsu, H. Nakayama, T. Hayashi and H. Matsuo R. Kawashima, "SCLP: Segment-oriented Connection-less Protocol for high-performance software tunneling in datacenter networks," in Proceedings of the 2015 1st IEEE Conference on Network Softwarization (NetSoft), London, 2015, pp. 18.

[32] J. Hamalainen and J. Rajala, "Connection-less packet data transmission in the signalling network infrastructure," in Proceedings of 1994 3rd IEEE International Conference on Universal Personal Communications, San Diego, CA, USA, 1994, pp. 511-515.

[33] Behrouz A Forouzan, Data Communications and Networking NY: McGraw-Hill, 5th ed. New York, 2012.

[34] T. Yonghui and R. Hu, "A Resolution for IGMP V3 Protocol Using Finite State Machine," in International Conference on Computer Science and Electronics Engineering, Hangzhou, 2012, pp. 517-520.

[35] P. Mérindol, B. Donnet, A. Pescapé and J. Pansiot P. Marchetta, "Quantifying and mitigating IGMP filtering in topology discovery," in IEEE Global Communications Conference (GLOBECOM), Anaheim, CA, 2012, pp. 1871-1876.

[36] Network Working Group of the IETF. (2006, January ) The Secure Shell (SSH) Protocol Architecture. [Online]. https://tools.ietf.org/html/rfc4251

[37] D. Gatzen, A. Panchenko and U. Meyer A. Egners, "Introducing SOR: SSH-based Onion Routing," in 26th International Conference on Advanced Information Networking and Applications Workshops, Fukuoka, 2012, pp. 280-286.

[38] W. Ryu and J. K. Choi K. W. Hong, "Telnet-based transport control," in 11th International Conference on Advanced Communication Technology, Phoenix Park, 2009, pp. 887-889.

[39] Y. Chai and W. Shi, "Access-enhanced hybrid routing protocol for hybrid wireless mesh network," in IEEE 9th International Conference on Communication Software and Networks (ICCSN), Guangzhou, 2017,pp. 138-141.

[40] Xiuwei Yang, Lin Zhang and Yanbo Zhang F. Wang, "Design of environmental monitoring system based on XMPP," in 2nd International Conference on Measurement, Information and 2nd International Conference on Measurement, Information and, Harbin, 2013, pp. 509-512.

[41] J. Wu, Z. Xia and J. Li L. Guo, "Proposed Security Mechanism for XMPP-Based Communications of ISO/IEC/IEEE 21451 Sensor Networks," IEEE Sensors Journal, vol. 15, no. 5, pp. 2577-2586, May 2015.

[42] S. Vinoski, "Advanced Message Queuing Protocol," IEEE Internet Computing, vol. 10, no. 6, pp. 87-89, Nov.-Dec 2006.

[43] S. Kim, K. Lim and S. Lee D. Pak, "Lowdelay stream switch method for real-time transfer protocol," in The 18th IEEE International Symposium on Consumer Electronics (ISCE 2014), JeJu Island, 2014, pp. $1-2$.

[44] Guocai Jia, Fuhong Lin, Chao Guo and 
Xiao Ma Lin Yao, "Service analysis and optimization program of LAN based on OPNET," in International Conference on Cyberspace Technology (CCT 2013), Beijing, China, 2013, pp. 431-437.

[45] A. Braga et al., "Implementation of a new propagation model for $5.8 \mathrm{GHz}$ systems in OPNET simulator," in 7th European Conference on Antennas and Propagation (EuCAP), Gothenburg, Gothenburg, 2013, pp. 954-957.

[46]nsns-users@isi.edu. Information $\mathrm{Sc}$ iences Institute. [Online]. https://www.isi.edu/nsnam/ns/

[47] F. Du and X. Fan, "Ant Colony Algorithm Study and Implementation Based on Ns2," in 8th International Symposium on Computational Intelligence and Design (ISCID), Hangzhou, 2015, pp. 633-636.

[48] S. Chang and S. Lee J. Jung, "Appropriate module configuration for vehicular networking using NS2 simulator," in
International Conference on Information and Communication Technology Convergence (ICTC), Busan, 2014, pp. 611-612.

[49] Inc Cisco Systems. Cisco System. [Online]. https://www.cisco.com/c/en/us/td/ docs/security/asa/asa-command-reference/I$\mathrm{R} / \mathrm{cmdref} 2 / \mathrm{p} 1 . h t m l$

[50] G. Predusca, L. D. Circiumarescu, N. Angelescu and D. C. Puchianu C. G. Dumitrache, "Comparative study of RIP, OSPF and EIGRP protocols using Cisco Packet Tracer," in 5th International Symposium on Electrical and Electronics Engineering (ISEEE), Galati, 2017, pp. 1-6.

[51] Z. Trabelsi and H. Saleous, "Exploring the Opportunities of Cisco Packet Tracer For Hands-on Security Courses on Firewalls," in IEEE Global Engineering Education Conference (EDUCON), Dubai,United Arab Emirates, 2019, pp. 411-418. 